\title{
TAME ARCS ON DISKS
}

\author{
JOSEPH MARTIN ${ }^{1}$
}

It is the goal of this note to show that each disk in $E^{3}$ contains a tame arc which intersects the boundary of $D$. In [1] Bing shows that each disk in $E^{3}$ contains many tame arcs. The reason that the arguments given in [1] do not show that each disk contains a tame arc intersecting the boundary is that a disk in $E^{\mathbf{3}}$ need not lie on a closed surface in $E^{z}$ [7]. This difficulty can be overcome using Bing's improvement of the "side approximation theorem" [2] and a theorem of Hempel [6].

Suppose that $D$ is a disk in $E^{8}$.

Lemma. If $D$ lies on a 2-sphere in $E^{3}$ then $D$ contains a tame arc which intersects both Int $D$ and $\mathrm{Bd} D$.

Proof. Let $S$ be a 2-sphere in $E^{3}$ containing $D$. It follows from [1] that for each positive number $\epsilon$ there exists a tame 2-sphere $S^{\prime}$ such that (i) $S \cap S^{\prime}$ contains a tame Sierpinski curve $X$ and, (ii) each component of $S-X$ is of diameter less than $\epsilon$.

Now if $\epsilon$ is chosen less than $\min \{\operatorname{diam} D, \operatorname{diam}(S-D)\}$ then $X$ must intersect both $D$ and $S-D$, and hence $\mathrm{Bd} D$. It follows that $D$ contains a tame arc which intersects both Int $D$ and Bd $D$. This establishes the lemma.

TheOREM. $D$ contains a tame arc which intersects both Int $D$ and Bd $D$.

Proof. Let $J_{1}, J_{2}, \cdots$ be a sequence of tame simple closed curves on $D$ such that if $D_{1}, D_{2}, \cdots$ are the disks on $D$ bounded, respectively, by $J_{1}, J_{2}, \cdots$ then $D_{i} \subset$ Int $D_{i+1}$ and $U D_{i}=\operatorname{Int} D$. The existence of these tame simple closed curves follows from [1]. It follows from a theorem of Hempel [6] that for each $i, D_{i}$ lies on a closed surface in $E^{3}$. This is because $D_{i}$ is interior to the larger disk $D_{i+1}$. Now, using this fact and repeatedly applying the results of [2] and the techniques of $[1]$, there exist tame disks $D_{1}^{\prime}, D_{2}^{\prime}, \cdots$ such that 1963.

Presented to the Society August 29, 1963; received by the editors September 7,

1 This paper was written while the author was a postdoctoral fellow of the National Science Foundation. 
(a) $\operatorname{Bd} D_{i}^{\prime}=J_{i}$,

(b) $D_{i} \cap D_{i}^{\prime}$ is a Sierpinski curve,

(c) $D_{i}^{\prime} \subset$ Int $D_{i+1}^{\prime}$, and

(d) $\mathrm{Cl}\left[\cup D_{i}^{\prime}\right]$ is a disk bounded by $\mathrm{Bd} D$.

The procedure for obtaining these disks is, roughly, as follows: a dense, null sequence of disks is removed from the interior of $D_{1}$ and each of these disks is replaced by a tame disk. The resulting tame disk is $D_{1}^{\prime}$. Then, disks are removed from the annulus on $D$ bounded by $J_{1}$ and $J_{2}$ and are replaced by tame disks to obtain $D_{2}^{\prime}$. This process is continued. Care is exercised in replacing disks with tame disks so that each of $D_{i}^{\prime}$ and $\mathrm{Cl}\left[\cup D_{i}^{\prime}\right]$ is a disk. It follows from a theorem of Gillman [4] that the disks which are removed at the $i$ th stage need not intersect $J_{i}$. For more details on this replacing process the reader is referred to [1].

Now let $D^{\prime}$ denote $\mathrm{Cl}\left[\cup D_{i}^{\prime}\right]$. Notice that $D \cap D^{\prime}$ is a Sierpiński curve which contains $\mathrm{Cl}\left[\cup J_{i}\right]$. Now $D^{\prime}$ is locally tame at each point of Int $D^{\prime}$ and it follows from [3] that there is no loss in generality in assuming that $D^{\prime}$ is locally polyhedral at each point of Int $D^{\prime}$. It follows from [5] that $D^{\prime}$ lies on a 2-sphere in $E^{3}$.

Now by the lemma there exists a tame arc $\alpha$ on $D^{\prime}$ which intersects both Int $D^{\prime}$ and $\mathrm{Bd} D^{\prime}$. Without loss of generality we may assume that $\alpha \cap B d D=\{P\}$. Let $\beta$ be an arc in $D \cap D^{\prime}$ having $P$ for one endpoint and such that $\beta-\{P\} \subset$ Int $D$.

Let $K$ be a subdisk of $D^{\prime}$ such that (i) $\alpha \cup \beta \subset K$, (ii) $K \cap B d D^{\prime}$ $=\{P\}$ and, (iii) $K$ is locally polyhedral except at $P$. Then there is a 2-sphere $S$ in $E^{3}$ such that $K \subset S$ and $S$ is locally polyhedral except at $P$. But $P$ lies on the tame arc $\alpha$ and it follows from [8] that $S$ is a tame 2 -sphere. Thus the arc $\beta$ is tame and satisfies the conclusion of the theorem. This establishes the theorem.

Notice that the arguments given actually show that the set of points of $\mathrm{Bd} D$ which are accessible by tame arcs from Int $D$ is dense in $\operatorname{Bd} D$.

CoRollary. If $\epsilon>0$ then there is a triangulation $T$ of $D$ such that (i) mesh $T<\epsilon$ and (ii) if $\sigma$ is a wild 1-simplex of $T$ then $\sigma \subset B d D$.

\section{REFERENCES}

1. R. H. Bing, Each disk in $E^{z}$ contains a tame arc, Amer. J. Math. 84 (1962), 583590.

2. - Improving the side approximation theorem, Abstract 603-72, Notices Amer. Math. Soc. 10 (1963), 453.

3. - Locally tame sets are tame, Ann. of Math. (2) 59 (1954), 145-158. 
4. David Gillman, Side approximation missing an arc, Amer. J. Math. 85 (1963), 459-476.

5. O. G. Harrold, H. C. Griffith and E. E. Posey, A characterization of tame curves in three-space, Trans. Amer. Math. Soc. 79 (1955), 12-34.

6. J. P. Hempel, Extending a surface in $E^{3}$ to a closed surface, Abstract 63T-65, Notices Amer. Math. Soc. 10 (1963), 191.

7. I. Kapuano, Sur les surfaces homeomor phes d un disque dans un $R^{2}, C$. R. Acad. Paris 256 (1953), 1229-1230.

8. E. E. Moise, Affine structures in 3-manifolds. VIII. Invariance of the knot types; local tame embedding, Ann. of Math. (2) 59 (1954), 159-170.

The Institute for Advanced Study

\section{SPECIAL $n$-MANIFOLDS WITH BOUNDARY ${ }^{1}$}

P. H. DOYLE AND J. G. HOCKING

By a K-R manifold we mean an $n$-manifold with boundary $M^{n}$ such that Int $M^{n}=E^{n}$ and $\mathrm{Bd} M^{n}=E^{n-1}$; Int $M^{n}$ and $\mathrm{Bd} M^{n}$ are the interior and boundary of $M^{n}$ respectively. Both Cantrell [2] and Doyle [3] have shown that for $n \neq 3$, each $K-R$ manifold is the product $E^{n-1} \times[0,1)$. But for $n=3$ there are infinitely many $\mathrm{K}-\mathrm{R}$ manifolds which are topologically distinct as pointed out in [4] and [5]. We will investigate certain properties of these manifolds with boundary.

Lemma 0. Let $M^{n}$ be a $K-R$ manifold. Then $M^{n}$ is the product $E^{n-1} \times[0,1)$ if each compact set in $M^{n}$ lies in a closed $n$-cell in $M^{n}$.

Proof. The proof is simple in that $M^{n}$ can be represented as a union of closed $n$-cells $\cup C_{i}$ where $C_{i} \cap \mathrm{Bd} M^{n}$ is an $(n-1)$-cell $D_{i}$ nicely imbedded in $\mathrm{Bd} C_{i}$ and $\mathrm{Bd} M^{n}, D_{i} \subset \operatorname{Int} D_{i+1}$ and $C_{i}-D_{i}$ CInt $C_{i+1}$, while $\left[C_{i+1}-C_{i}\right]-$ is an $n$-cell. One can then construct a homeomorphism of $M^{n}$ onto a copy of $E^{n-1} \times[0,1)$.

LEMMA 1. Let $M^{n}$ be an n-manifold with boundary. If $C$ is a compact set in $M^{n}$ such that $C \cap B d M^{n}$ lies in an open $(n-1)$-cell in $\mathrm{Bd} M^{n}$, then there is a pseudo-isotopy $h_{t}$ of $M^{n}$ onto $M^{n}$ such that $h_{1}(C) \subset F \cup C^{\prime}$, where $F$ is a fiber in a collar about $\mathrm{Bd} M^{n}$, and $C^{\prime}$ is a compact set in Int $M^{n}$.

Received by the editors August 4, 1963.

1 The work was done under National Science Foundation Grant GP-31. 